\title{
Reflexión sobre los Modelos para el Estudio de los Brotes de Rabia Humana por Murciélago
}

\author{
Comments on Models for Studying Human Rabies Outbreaks from Bats
}

\author{
Maria Cristina Schneider ${ }^{1}$
}

SCHNEIDER, M. C. Comments on Models for Studying Human Rabies Outbreaks from Bats.
Cad. Saúde Públ., Rio de Janeiro, 11 (2): 291-304, Apr/Jun, 1995.

This paper analyzes which of the possible public health models could contribute to an understanding of human rabies outbreaks transmitted by bats. First, we present a short review of the most common pertinent models. Second, we present an investigation of a human rabies outbreak transmitted by bats based on the socio-epidemiological model. The same outbreak is analyzed again using the complex system theory as a theoretical framework, as both a theoretical-practical exercise seeking an approximation to this theory and a model to study this problem.

Key words: Rabies; Bats; Complex System Theory; Public Health

\section{INTRODUCCION}

Al mismo tiempo que se está logrando un mejor control del ciclo urbano de la rabia en Brasil, existe un aumento importante de los casos de rabia humana trasmitidos por murciélagos, pasando a ser la segunda especie, después de los perros, responsable por los casos humanos. En 1980, fueron registrados 168 casos humanos, de los cuales sólo uno había sido trasmitido por murciélagos, representando el $0.6 \%$ del total de casos. Desde 1985 viene aumentando el número de muertes como resultado de rabia transmitida por vampiros. En 1990, 11 de los 73 casos registrados en el país fueron relacionados a murciélagos hematófagos, representando el $15 \%$. La mayoría de los brotes que ocurrieron en 1990 fueron en lugares en donde muchas personas fueron agredidas. Generalmente estos casos acontecen en áreas de difícil acceso, localizándose algunos de ellos en la región amazónica. En los brotes investigados se puede sugerir una posible asociación entre el tipo de proceso productivo local y cambios

\footnotetext{
' 14306 Night Hawk Way. North Potomac, MD, 20878, USA.
}

importantes en él, con la ocurrencia de los casos (Schneider, 1991).

¿Qué está ocurriendo en determinados lugares para que los murciélagos hematófagos se alimenten de sangre humana en gran escala, provocando la transmisión de rabia a las personas?

Los modelos de salud pública más utilizados en las últimas décadas (Leavell \& Clark, 1965; Mac Mahon \& Pugh, 1970; Susser, 1973; Pérez Tamayo, 1974; San Martín, 1975) no siempre satisfacen totalmente con sus explicaciones el entendimiento del problema. Segundo Frenk (1979: 133), menciona que una de las incertidumbres en el saber sobre la salud es "la falta de comprensión sobre los factores que determinan la salud y, la enfermedad y sobre la forma en que se interrelacionan".

La búsqueda por construir nuevos modelos o por conocer otros modelos existentes no hegemónicos es fundamental para que se amplíe el conocimiento sobre los procesos de salud-enfermedad.

Existe suficiente conocimiento sobre el aspecto biológico de los murciélagos hematófagos con relación a la transmisión de rabia humana (Greenhall \& Scmidt, 1989; Uieda, 1987; Baer, 1975). El problema a ser 
estudiado no es solamente biológico, por ejemplo puede tener determinados factores biológicos, como las condiciones naturales de abrigo y fauna silvestre para el mantenimiento de esta especie, y la circulación del virus en el ciclo silvestre de la enfermedad, pero también tiene factores sociales y económicos bastante evidentes.

El vampiro y la rabia transmitida al ganado vacuno existen en todo el territorio brasileño. La rabia humana trasmitida por murciélagos ocurre esporádicamente, casi siempre en determinadas situaciones que involucran ciertos tipos de procesos productivos y condiciones de vida. Es fundamental pero no suficiente el análisis social y económico de los lugares donde están ocurriendo los brotes.

Nos parece que la clave para el entendimiento de este problema es terminar con la dicotomía entre los elementos biológicos (o naturales) y los no biológicos (o sociales). El objetivo de esta reflexión es cómo hacer este análisis situacional, teniendo una visión integrada del todo y no de partes separadas.

Es fundamental escoger el marco teórico que mejor contribuya al entendimiento de nuestro problema, permitiendo de esta forma proponer mejores intervenciones en el intento de disminuir el número de casos de rabia humana por vampiros. Un ejemplo de lo anterior sería desarrollar un modelo epidemiológico para definir situaciones de riesgo, con el fin de comenzar con las acciones de control antes que se presenten los casos.

El modelo más utilizado para estudiar la rabia ha sido el epidemiológico clásico. Algunos estudios sobre el mismo tema, han sido desarrollados por nosotros con base en el modelo epidemiológico social, aunque también pueden ser utilizados otros modelos.

La teoría de los sistemas complejos (García, 1986) nos parece bastante adecuada para fundamentar el estudio propuesto, puesto que tiene la visión integradora que buscamos.

Este ensayo pretende reflexionar sobre cuál modelo contribuida más para el entendimiento de nuestro problema. Serán abordadas las ventajas y limitaciones de los modelos en salud pública más conocidos que pudieran ser utilizados para analizar los brotes.

En un segundo momento y como un ejemplo del marco teórico que venimos utilizando, será presentada la investigación de un brote de rabia humana transmitido por murciélagos, con base en el modelo epidemiológico social. Este mismo brote será analizado con un enfoque sistémico, donde también serán incluidos algunos conceptos de la teoría de los sistemas complejos. Este ejercicio teórico-práctico nos permitirá tener una primera aproximación con esta teoría, con el fin de ver qué tan útil puede ser para entender mejor el problema que estamos tratando.

\section{LA SELECCION DE UN MODELO PARA ESTUDIAR LOS BROTES DE RABIA HUMANA POR URCIELAGOS}

La rabia ha sido una enfermedad bastante analizada desde tiempos inmemoriales. Como otras enfermedades, ha sido estudiada y entendida de acuerdo con el pensamiento hegemónico que en su momento histórico explicó los procesos salud-enfermedad, como ha sido observado por Schneider \& Burgoa (1994).

Para este ensayo nos interesa los modelos vigentes actualmente, que podrían explicar lo que está ocurriendo en determinados lugares para que surjan brotes de rabia humana por murciélagos.

La investigación de la rabia podría ser dividida conforme al cuadro desarrollado por Frenk (1992), teniendo ejemplos de investigaciones para todas las clasificaciones (Figura 1).

Desde nuestro punto de vista, el estudio propuesto parte de una investigación por consecuencias que serían los brotes de rabia. Por medio de estas investigaciones se intentaría desarrollar un modelo epidemiológico para definir situaciones de riesgo que pasaría posteriormente a una 
FIGURA 1. Integración entre las Investigaciones con Relación al Objeto de Estudio

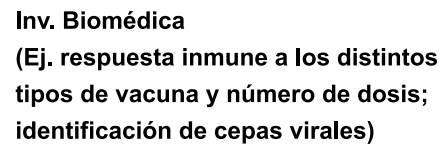

(Ej. respuesta inmune a los distintos tipos de vacuna y número de dosis;

identificación de cepas virales)

Investigación

en salud

(Ej. rabia)

Inv. Clínica
(Ej. cuadro clínico
de la enfermedad)

Inv. Salud Pública

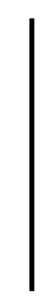

Inv. Epidemiológica

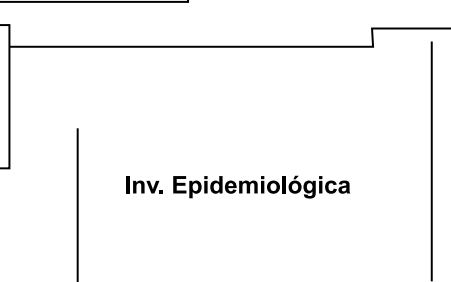

Inv. Sistema de Salud

Inv. Sistema de Salud

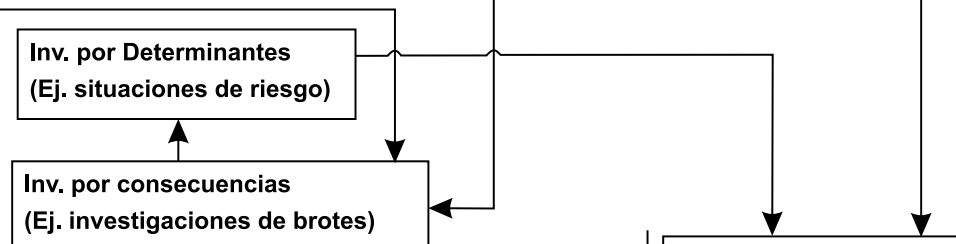

Inv. Sistema de Salud
(Ej. Calidad del tratamiento)

Inv. en Recursos para la Salud

(Ej. Accesibilidad al tratamiento)

Inv. en Políticas de Salud

(Ej. El control de la Rabia como prioridad

del Ministerio de Salud)

Fuente: Modificado de Frenk (1992). 
investigación por determinantes. El modelo desarrollado sería propuesto al Ministerio de Salud en Brasil para ser utilizado en los servicios (Programa Nacional de Profilaxis de la Rabia - PNPR), de tal manera que sirviera como una base para la toma de decisiones al usar diferentes estrategias para las áreas de riesgo. Una investigación de los servicios pudiera ser el llevar a cabo un mapeo de los sitios de mayor riesgos de rabia en Brasil, al mismo tiempo que la implementación de acciones de control del ciclo silvestre por el Ministerio de Salud pasaría a ser una política de salud. Para contribuir a los estudios de los brotes sería importante el apoyo de investigaciones biomédicas sobre las cepas virales de los casos ocurridos e investigaciones clínicas sobre sintomatología, período de incubación, etc., de los casos. Para proponer las acciones de control también sería necesario el apoyo de investigaciones biomédicas para definir el tipo de vacuna y el número de dosis más indicado. Así mismo, a través de la investigación de los servicios de salud debería ser verificada la calidad del tratamiento y la accesibilidad de las personas agredidas, demostrando la interrelación entre los distintos tipos de investigación para el mismo objeto de estudio (Figura 1).

La complexidad del problema es bastante grande, aunque por el momento sólo nos interesa la primera parte, que es el entendimiento sobre los brotes.

El modelo buscado, como se mencionó anteriormente, debería analizar de forma conjunta los elementos biológicos y sociales, de manera tal que permita no sólo conocer los factores más importantes relacionados con los brotes, sino establecer también las relaciones entre los elementos, lo cual nos llevaría a entender los procesos que determinan los brotes.

Otra consideración importante sería que el modelo utilizado tenga una visión histórica, porque probablemente las causas de lo que está ocurriendo no empezaron en el momento de la investigación. Es básico poner en un contexto general el problema, con el fin de conocer los factores externos que pudieran estar influyendo.

La investigación epidemiológica de la rabia en general es desarrollada en base al modelo epidemiológico clásico (Mac Mahon \& Pugh, 1970; Lillienfeld \& Lillienfeld, 1980), y la mayoría de las veces de forma descriptiva. Varios brotes sobre rabia humana transmitida por murciélagos fueron investigados en Brasil (Konolsaisen et al., 1991; Schneider, 1991; Pereira-Filho, 1990) y en otros países de América Latina (López, 1991) con este enfoque. Para el análisis de este problema y de acuerdo con lo que este modelo permite, nos parece suficiente la investigación de servicios; sin embargo, nos parece limitado para tener una explicación más profunda de lo que realmente está ocurriendo en estas áreas.

El otro modelo que se aplicaría a este tipo de investigación sería el ecológico (Dubos, 1975; Susser, 1973). En la revisión hecha no fueron encontrados brotes de rabia investigados con este modelo. Existen trabajos en salud pública veterinaria (Astudillo et al., 1986; Rosemberg, 1986) que utilizan de forma conjunta el enfoque dado por el modelo ecológico complementado por el sociomédico, corriente marxista (Laurell, 1989; Breilh \& Granada, 1986) que nos parece bastante interesante.

El modelo sociomédico y el epidemiológico social (Castellano, 1987) parecen tener varias de las características buscadas, pero para nuestro objeto de estudio creemos que tienen un inconveniente. Lo anterior se refiere a que los dos modelos desarrollan con mucho detalle el análisis de los aspectos sociales, en detrimento de aquellos de otro tipo. Sin duda lo social desempeña un papel determinante en este estudio, pero los aspectos biológicos también deben ser mejor conocidos. Todavía hay muy pocas investigaciones con relación a hábitos alimentarios y de otros tipos de comportamiento de los murciélagos desde el punto biológico, como la circulación del virus de la rabia en el ciclo silvestre en Brasil.

Algunos estudios en el área de zoonosis (Schneider, 1990; Galvão, 1988; Silva, 1985) fueron realizados con este enfoque, utilizando de forma conjunta la teoría de la organización social del espacio (Moraes \& Costa, 1987; Santos, 1986) y la teoría de los focos de 
Pavlovsky (sal.), en la cual el estudio de los aspectos biológicos se complementaría más.

Desde nuestro punto de vista y con relación al objeto de estudio pretendido, el modelo epidemiológico social y el sociomédico, darían explicaciones semejantes, debido a esto no se van a discutir los dos modelos por separado. Segundo Frenk (1992), menciona que el modelo epidemiológico social tiene como perspectiva de análisis, la enfermedad y el sociomédico los determinantes de la salud; no obstante, nos parece difícil clasificar los estudios realizados por nosotros en Brasil dentro de un modelo específico, porque nos parecen ser estudios más eclécticos, utilizando un poco de los distintos modelos. Sin embargo, sin estar totalmente correcto, vamos a llamar al enfoque dado a nuestro estudio sobre el brote de Boca da Mata como epidemiológico social, puesto que hay una mayor identificación con los autores del modelo sociomédico, corriente marxista. Esta reflexión se trata sobre el modelo que mejor explique por qué están ocurriendo brotes de rabia humana por murciélagos. Por lo tanto, en esta reflexión seguiremos comparando un brote investigado con el enfoque epidemiológico social y el mismo brote investigado hipotéticamente con el enfoque sistémico, intentando conocer las nuevas aportaciones que la teoría de los sistemas complejos podría dar.

\section{EL BROTE DE BOCA DA MATA: ENFOQUE EPIDEMIOLOGICO SOCIAL}

En mayo de 1986 fue comunicado a la Coordinación Nacional del PNPR el surgimiento de dos casos de rabia humana transmitidos por murciélagos, en dos niñas de nueve años de edad, primas, residentes en la misma casa en una industria para el beneficio de la caña de azúcar en el Municipio de Boca da Mata, en la zona de la Mata da Alagoas, Brasil.

La Coordinación Estatal del Programa, juntamente con la Nacional y un órgano de la Agricultura (EMATER), estuvieron en el lugar para tomar las acciones pertinentes e investigar el caso.
El área visitada comprende dos beneficios de caña vecinos, divididos por un pequeño río, existiendo una colónia con aproximadamente 40 familias de un lado y una colónia de otro beneficio de caña, con más de 57 familias, pero, éstas presentaban «condiciones de mordida» acentuadamente mejores.

La región puede ser descrita como una de las tantas áreas del noreste con grandes extensiones de monocultivo de exportación, en este caso, la caña de azúcar. La región sufrió un gran desmonte en los últimos 20 años, período en el cual todo Brasil tuvo un incentivo muy importante para la expansión de la agricultura y mecanización del campo.

Al hacer un análisis del proceso histórico brasileño entre los años 50 hasta 1967, se encuentra a la modernización de la agricultura como un aspecto muy importante. En este período, se establecieron las bases para la producción de bienes de capital (maquinaria y equipamiento agrícolas) (Moreira, 1982).

La política de créditos rural se inició conjuntamente, proporcionando las bases financieras para los cambios, al mismo tiempo en que eran derrotadas las propuestas de reforma agraria.

En el caso de la caña de azúcar, además del incentivo que existía para el plantío de un monocultivo de exportación, el interés por estos productos aumentó todavía más ante la crisis del petróleo que surgió en los años 70s, en el momento en que Brasil pasó a producir combustible para automóviles a base de alcohol, utilizando para dicho fin a todo el país.

El aumento en la demanda de materia prima requirió. cambios tecnológicos. Estos cambios acarrearon la disminución en el número de beneficios de caña, esto es, el fortalecimiento de los grandes beneficios de caña mecanizados y disminuyendo los pequeños productores, que a su vez, ya estaban divididos por la política de crédito rural (Moreira, 1981).

El interés por el aumento de la producción de caña de azúcar condujo a la necesidad de una mayor área cultivada; de la modernización de la tecnología utilizada; al requerimiento de un menor número de 
trabajadores, principalmente en los meses intermedios entre el plantío y la cosecha; además de otros factores; también provocó el fin de gran parte de las colónias y en consecuencia un éxodo rural-urbano.

Aquellos trabajadores que ya no estaban estables en haciendas, pero sí eran bolas frías residentes en favelas en las periferias de las ciudades vecinas, formaron un ejército industrial de reserva a la disposición del capital.

Los que permanecieran viviendo en las haciendas, perdieron el sistema de morada (significa tener una casa para el empleado y su familia en la finca), que permitía al trabajador un espacio de tierra para el plantío de alimentos de subsistencia y crías de animales a pequeña escala, cambiando en gran medida las condiciones de reproducción de estos grupos sociales y sus familias. Actualmente al igual que el régimen de meieiro (significa que una persona utiliza para plantar la tierra de otro individuo y las ganancias se dividen entre los dos) o perceiro en el cual el propietario recibe parte de la producción casi no existe, sólo un costado de cerro o áreas no productivas que no sean cedidas. Hoy día la caña de azúcar va hasta casi la ventana de la casa del trabajador.

A partir de la pérdida del sistema de morada y de meieiro quisiéramos regresar al caso de Boca da Mata. Es evidente que estos procesos no se dan de manera uniforme en todo el Brasil y todavía no podemos precisar específicamente cómo van ocurriendo en aquella región. Al platicar con los trabajadores más antiguos, podemos decir que la pérdida de ese espacio de tierra que tenían para el plantío de las necesidades caseras y la orden para la eliminación de los animales que poseían, ocurrió hace ocho años aproximadamente.

Es más o menos de esa época para acá, que los moradores de una de las haciendas relatan la aparición de personas agredidas por murciélagos durante el sueño. Un levantamiento de las personas agredidas en los últimos tiempos, realizado por una asistente social de uno de los beneficios de caña, después del surgimiento del primer caso de rabia en la región, enviaron cerca de 40 personas para tratamiento. Al platicar con los agricultores también nos fue referido el miedo al tratamiento y la no credibilidad de que podrían enfermar, y otro aspecto muy importante a considerar fue la pérdida del día de trabajo debida al tratamiento. Durante la investigación, los moradores de la hacienda en condiciones más precarias, relataron un número bastante grande de personas agredidas, mucho más que los 40 anteriormente encontrados.

Los pocos animales existentes en el lugar son constantemente agredidos por murciélagos, viniendo a presentar en algunos casos sintomatología compatible con la rabia. La familia de las víctimas tenía una cabra, que era constantemente atacada por hematófagos. Con la pretensión de salvar una de sus pocas fuentes de proteína animal, decidieron meterla a la casa para que durmiera. La casa era muy pobre, construida de pau-a pique (significa una construcción hecha con barro, principalmente) con aberturas arriba, lo cual permitía la entrada de vampiros, atraídos por la cabra que murió con síntomas de anemia.

Al visitar el lugar, son visibles las diferencias existentes entre los dos beneficios de caña. La primera, donde no hay relatos de agresión por murciélagos, ofrece relativamente buenas habitaciones para sus trabajadores, son casas de alvenaria, con techos de barro, energía eléctrica, lavandería pública y otros beneficios que no siempre se encuentran en la región.

El beneficio de caña no permite que los trabajadores mantengan un plantío de subsistencia, puesto que produce parte de la alimentación básica y la vende al agricultor por medio de un sistema de crédito. Esta deuda se va acumulando, llegando a valores muy altos que impiden que el trabajador renuncie a trabajar en el beneficio de caña. Los agricultores que llegan a residir dentro de las haciendas pasan por una rigurosa selección, muchas veces son hijos de antiguos trabajadores del beneficio de caña. Una vez que el agricultor se vuelve de carteira (significa una persona que tiene un contrato firmado con el empleador en un documento especial de trabajo que se utiliza en Brasil) raramente sale del beneficio de caña.

Las condiciones de vida de los empleados del beneficio de caña del otro lado del río, 
donde ocurrieron los casos, son mucho peores: las casas están en hileras, hechas de pau-a-pique, tejado de paja con aberturas y sin luz eléctrica.

Los dos beneficios de caña cuentan con asistencia médica, psicológica y de servicio social. No podemos opinar sobre la calidad de los servicios, pero el acceso depende del transporte del beneficio de caña debido a las grandes distancias dentro de las haciendas. No existe en la región un tratamiento profiláctico humano contra la rabia; lo hubo durante algún tiempo en el puesto de salud de la Secretaría de Salud, en la cabecera del municipio, pero debido a problemas diversos ha sido desactivado. Por lo tanto, para realizar el tratamiento es necesario irse a la capital del Estado, Maceió, que se encuentra a $80 \mathrm{Km}$, sin contar la distancia de las colónias hasta la carretera. En este brote después de la muerte de las niñas, el beneficio de caña proporcionó transporte para hacer el tratamiento, pero esta situación no es la usual.

Quisiéramos señalar que el pago del trabajador de caña de azúcar en el beneficio de caña visitado es por día de trabajo, en el caso que no se presente por estar enfermo, recibe solamente un pequeño porcentaje. Como el sueldo ya de por sí es muy bajo y la familia normalmente numerosa, se imposibilita a un jefe de familia a realizar un tratamiento de 13 días conforme se propone. La persona tiene que optar entre dejar pasar hambre a su familia debido a la reducción de casi la mitad de su sueldo o correr el riesgo de morir de una enfermedad rara para la localidad.

Fueron realizadas dos capturas de murciélagos en el área, en épocas distintas. En la primera luego después de la muerte de las niñas, fueron capturados 20 murciélagos, de los cuales cinco fueron hematófagos.

Los murciélagos fueron tratados con anticoagulante (warfarina) y soltados nuevamente, para que pudiesen provocar la muerte de otros murciélagos, además de la suya. Fue calculado que debió haber ocurrido en la región, la muerte de otros 100 a 150 hematófagos. Solamente uno de estos vampiros fue enviado para examen de laboratorio, dando resultado negativo para la pesquisa del virus de la rabia.

En la segunda captura, tres meses después de la primera, los moradores no relataron más agresiones por vampiros. Esta vez debido al mal tiempo, fueron capturados solamente seis murciélagos, siendo uno hematófago. Todos fueran negativos para la pesquisa del virus de la rabia. Todos los perros de las dos colónias vecinas y de zonas aledañas fueran vacunados.

Nuestra conclusión es que, la pérdida del sistema de morada y meieiro acarreó la casi desaparición de los animales domésticos (de interés económico) que existían en el área; en cuanto a la población de murciélagos debe haberse mantenido igual, ya que tienen una vida media de 12 años. Estos se vieron en la necesidad de alimentarse más frecuentemente de sangre humana, como una opción adecuada.

Esta situación se agravó por la falta de tratamiento profiláctico accesible, por el sistema de pago por día de trabajo que no permite el tratamiento durante 13 días como se propone y por el desconocimiento de la gravedad de esta situación nueva que se presentó en aquella región; todo lo anterior, determinó el surgimiento de dos casos de rabia humana y la exposición al riesgo de muchas otras personas.

\section{EL BROTE DE BOCA DA MATA: ENFOQUE SISTEMICO}

Este ensayo no tiene la pretensión de analizar una teoría y ni sería posible en el tiempo en que éste se desarrolló. Hacer un trabajo epistemológico necesita mucho estudio, empezando por conocer las bases epistemológicas que contribuyeron en la elaboración de la teoría analizada. Nuestro objetivo es de apenas tener el primer contacto con la teoría de los sistemas complejos (García, 1986), a través de lectura de parte de la bibliografía existente, intentando esbozar algunos de los principales conceptos a través de referencias.

Por las razones anteriores nos pareció interesante hacer un ejercicio teórico-práctico 
para desarrollar un estudio hipotético con base en la teoría de sistemas complejos. La tentativa de aplicar los conceptos de la teoría a un problema conocido, permitiría tener una mejor idea de cómo sería el estudio de un brote de rabia con enfoque sistémico. Esto no significa que las hipótesis propuestas sean correctas o que la teoría haya sido bien entendida, es apenas un ejercicio de pensamiento sistemático.

Vamos utilizar el brote de Boca da Mata como escenario por ser una realidad conocida y porque nos da la posibilidad de hacer una mejor comparación.

Como un primer abordaje a la teoría podríamos decir que puede ser aplicada al estudio de un ecosistema natural que ha sufrido la acción del hombre, ya sea por medio de la explotación de sus recursos renovables o no renovables (agrosistemas e industrias extractivas); o bien, por la instalación de asentamientos humanos de diversos tipos, incluyendo las grandes urbanizaciones y las obras de infraestrutura (García, 1986).

El objetivo de esta teoría puede definirse, en términos generales, como una propuesta para abordar el estudio de un «sistema global», que sería el conjunto de los elementos que intervienen en los procesos arriba mencionados (procesos económicos y políticos a ellos asociados), con sus partes o factores constitutivos, sus interrelaciones y sus interacciones con los demás sistemas (García, 1986).

La interpretación de un gran número de problemas es posible sólo cuando se comprenden plenamente las interacciones entre el medio físico, el sistema productivo y la organización socioeconómica de las fuerzas productivas. Esto requiere distinguir cuidadosamente la función de todas las partes y la estructura del sistema global (García, 1988a).

Hacer una investigación con enfoque sistémico es como estudiar una parte de la realidad que incluye aspectos físicos, biológicos, sociales, económicos y políticos (García, 1986). El investigador deberá definir qué parte de la realidad será ésta, y dependerá a su vez del objetivo de la investigación.
"El punto de partida está dado por el marco epistémico, que establece el tipo de pregunta - o conjunto coherente de preguntas - que significan la orientación general de investigación. En general, es posible formular una pregunta básica, con un conjunto de subpreguntas. Dada dicha pregunta conductora, la selección de los componentes del sistema (es decir, los elementos, los límites del sistema y sus interrelaciones, tanto internas como externas) es guiada por el grado de relevancia que éstas tengan con respecto a aquella" (García, 1986: 53)

Empezamos entonces por la pregunta conductora: ¿Qué ocurrió en Boca da Mata para que los murciélagos hematófagos se alimenten de sangre humana en gran escala, provocando la transmisión de rabia a dos niñas?

A partir de la pregunta conductora se pueden proponer algunas hipótesis para intentar definir el sistema, las cuales deberían ser comprobadas en el campo.

Los elementos del sistema suelen ser unidades también complejos (subsistemas) que interactúan entre sí (García, 1986). Entre los subsistemas existen flujos, que son las relaciones que forma la estructura del sistema. Un sistema también tiene flujos de entrada y salida con su contorno, que a su vez es creado por los factores externos que influyen en el sistema. Las condiciones del límite van a ser dados por los flujos entre los subsistemas y el contorno.

\section{Definición de los Elementos (Subsistemas) del Brote}

F - Físico

$\mathrm{P}$ - Agroproductivo

S - Socioeconómico

L - Servicios de Salud

Lo que aquí llamamos «sistema productivo» es, obviamente, un subsistema del sistema total que está integrado, además, por el sistema físico (suelo, agua, clima, 
relaciones ecológicas naturales) y el subsistema socioeconómico (estructura social, condicionantes económicas, factores políticos). Como ningún sistema está aislado en el tiempo y en el espacio, el estudio del sistema total requiere un análisis de lo que en otras publicaciones hemos llamado las "condiciones de contorno". Esto significa, que además de estudiar las interacciones entre los subsistemas, debemos considerar las interacciones del sistema total con el contexto en el cual está inmerso (García, 1988b). Para el brote analizado podríamos sugerir que "los factores externos que constituyen los cambios de las condiciones de contorno" (García, 1988b: 33), serían: Políticas de crédito, Proyecto Pro-alcohol, Sistema Nacional de Salud, Programa Nacional de Defensa Animal y posiblemente otros. Los factores externos que impactan una determinada realidad, en el caso Boca da Mata van a crear las condiciones de contorno.

\section{Condiciones de Contorno del Brote}

\section{Flujos de Entrada}

C1- Incentivo al plantío de monocultivo (caña)

C2 - Demanda del producto para combustible

C3 - Mecanización del campo

C4 - Fuerza de trabajo

C5 - Políticas de salud en aquel local

C6 - Políticas agropecuarias en aquel local

\section{Flujos de Salida}

S1 - Producto (azúcar y alcohol)

S2 - Emigración

La estructura del sistema está determinada por el conjunto de relaciones y no por los elementos. El sistema debe incluir aquellos elementos entre los cuales se han podido detectar las relaciones más significativas. Los otros elementos quedan «afuera» y las interrelaciones entre ellos y los elementos que quedan dentro, determinan las condiciones en los límites (García, 1986).

\section{Interrelaciones entre los Subsitemas del Brote}

a. Relaciones FP (del subsistema físico sobre el subsistema agroproductivo):

FPl - Condiciones edafológicas y climáticas para el cultivo de la caña

FP2 - Condiciones naturales relacionadas con la presencia de murciélagos

b. Relaciones PF (del subsistema agroproductivo sobre el subsistema físico):

$$
\begin{aligned}
& \text { PFl- Ampliación de la frontera } \\
& \text { agrícola (desmonte) } \\
& \text { PF2 - Control de la población de } \\
& \text { murciélagos (Programa de } \\
& \text { Defensa Animal del } \\
& \text { Ministerio de Agricultura } \\
& \text { contra la rabia en ganado } \\
& \text { vacuno) }
\end{aligned}
$$

c. Relaciones SP (del subsistema socioeconómico sobre el subsistema agroproductivo):

$$
\begin{aligned}
& \text { SP1 - Tenencia de latifundios } \\
& \text { SP2 - Condiciones de trabajo }
\end{aligned}
$$

d. Relaciones PS (del subsistema agroproductivo sobre el subsistema socioeconómico):

\section{PS1 - Tenencia del pátio}

e. Relaciones SL (del subsistema socioeconómico sobre el de servicios de salud):
SL1 - Conocimiento sobre el riesgo de rabia por murciélagos


SL2 - Búsqueda del tratamiento contra la rabia

f. Relaciones LS (del subsistema servicios de salud sobre el socioeconómico):
LS1 - Accesibilidad a los servicios de salud

LS2 - No aplicación del tratamiento
LS3 - Aplicación del tratamiento de forma inadecuada

g. Relaciones LF (del subsistema servicios de salud sobre el físico):

LFl - Control de población de murciélagos (acción conjunta con agricultura o individual cuando agricultura no lo hace)

Otro concepto importante para el entendimiento de los sistemas complejos es el relacionado con los niveles de procesos, los cuales pueden ser conocidos a través de las siguientes citas:
"El nudo central del análisis de la dinámica de los sistemas es el estudio de procesos. Los procesos describen los cambios que tienen lugar en el sistema. Pero ello requiere efectuar una cuidadosa distinción entre niveles de procesos, así como niveles de análisis"

(García, 1986: 59)

"Los procesos de primer nivel (o básicos) constituyen, generalmente, el efecto local, sobre el medio, físico o sobre la sociedad que lo habita y lo explota, de procesos más amplios que tienen lugar en otros lugares. Normalmente son empíricos, donde se busca algo en el terreno"

(García, 1986: 59)

"Un segundo nivel, que llamaremos meta procesos, corresponde a procesos más generales que gobiernan o determinan los procesos de primer nivel. Los metaprocesos pueden a su vez estar determinados por procesos de tercer nivel"

(García, 1986: 60)

FIGURA 2. Relaciones Estructurales en el Sistema de Boca da Mata

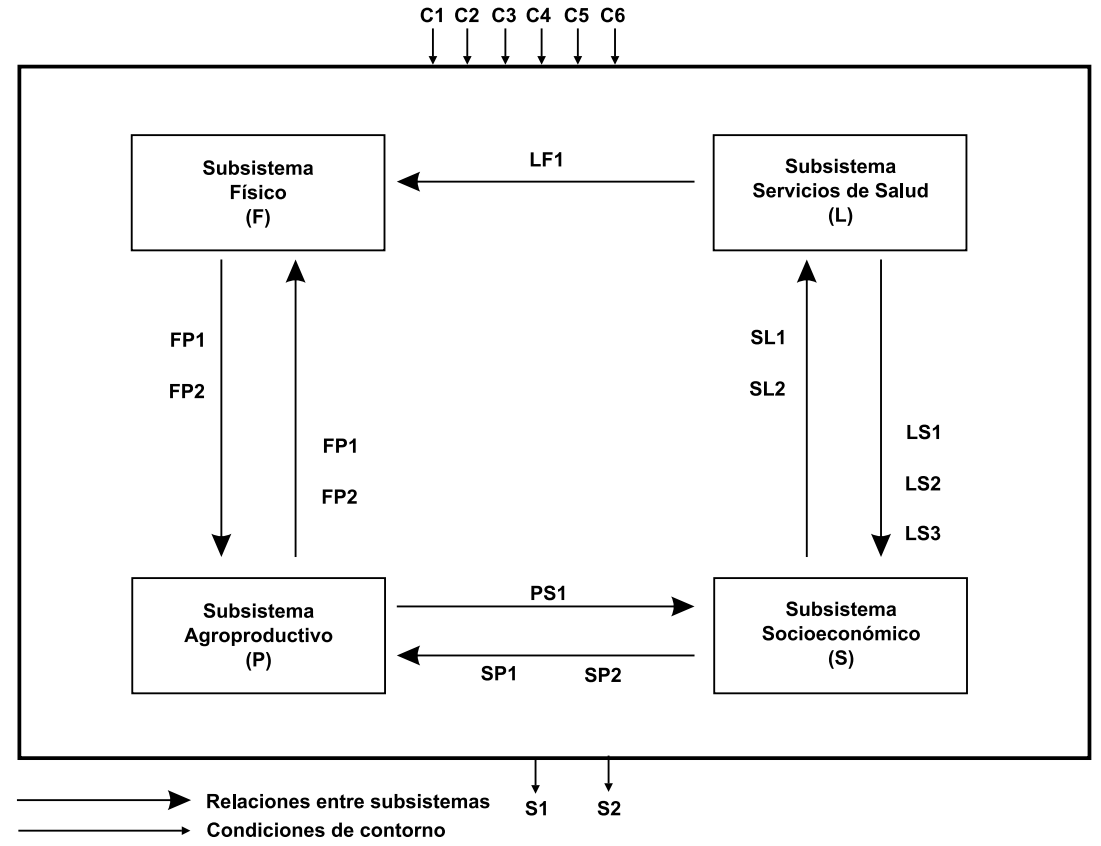

Fuente: Modificado de García (1988a) 


\section{Procesos en el Brote}

\section{Primer Nivel}

La cancelación de los pátios donde los trabajadores tenían animales domésticos para su consumo y transporte, así como pequeñas sembradíos de subsistencias. Esta prohibición provocó la brusca retirada de gran parte de los animales domésticos de la región que servían de fuente de alimentación para los murciélagos.

\section{Segundo Nivel}

El aumento del área cultivada de caña de azúcar, debido a una necesidad mayor por el producto industrializado en forma de alcohol. Modernización y equipamiento de los beneficios de caña para el procesamiento del alcohol.

\section{Tercer Nivel}

La crisis internacional del petróleo, que propició la búsqueda de formas alternativas de combustible como el Proyecto Pro-alcohol, que contribuiría a disminuir la falta de combustible en el país y favorecería a los grandes agricultores de caña de azúcar en el país, y que representan además a un sector bastante fuerte en la política nacional.

"En un estudio de la dinámica de un sistema es necesario analizar su historia. El período durante el cual se estudia la evolución depende de la naturaleza del sistema y de lo que hemos llamado 'la pregunta conductora' de la investigación” (García, 1986: PP)

\section{Escala Temporal del Brote}

La escala temporal a ser utilizada podría ser a partir de la creación del Proyecto Proalcohol en 1970, cuando empezó una gran movilización en esta área para aumentar la producción de caña de azúcar.

La política de mecanización del campo en Brasil empezó en los años 50, siguiendo posteriormente el incentivo para el plantío de monocultivos de exportación. No obstante, para esta región creemos que fue más significativo el cambio a la utilización de alcohol, como combustible para automóviles en Brasil.

“Todo sistema abierto (autoorganizado) está sometido a perturbaciones que pueden ser de muy diversas escalas. Ellas pueden ser de carácter exógeno (las cuales se traducen en modificaciones de las modificaciones de contorno) o de carácter endógeno (modificaciones de algunos de los parámetros que determinan las relaciones dentro del sistema). Si para cierta escala de perturbaciones, estas modificaciones oscilan dentro de ciertos límites sin alterar la estructura del sistema, diremos que el sistema es estable con respecto a dicha escala de perturbaciones. En estos casos, las perturbaciones son amortiguadas o incorporadas al sistemá. Cuando no ocurre ninguna de ambas alternativas, el sistema no puede «absorber» la perturbación. El sistema se torna inestable y ocurre una disrupción de su estructura. La evolución de un sistema después de la inestabilidad puede variar. Si la inestabilidad es desencadenada por una modificación en el contorno, puede ser respecto a cierto tipo de perturbaciones. $L$ a inestabilidad está a su vez asociada a los procesos de desestruturación y reestruturación del sistema. Son estos procesos y no la estructura misma, quienes constituyen el objetivo fundamental del análisis. Se trata pues, de un estudio de la dinámica del sistema y no del estudio de un estado en un momento dado" (García, 1986: 64)

"Los sistemas complejos requieren una concepción específica de la investigación interdisciplinaria y del análisis sistémico. La investigación interdisciplinaria es insistentemente preconizada por las instituciones de educación superior, poco practicada y frecuentemente confundida con trabajos multidisciplinarios. La investigación interdisciplinaria se basa, sin duda, en una metodología de trabajo en equipo multidisciplinario, pero se apoya, además, en un marco conceptual cuya fundamentación es epistemológica” (CINVESTAV, sal.: 07) 
"La visión sistémica aplicada a fenómenos complejos, sólo puede resultar de un trabajo que se plantee desde el inicio como una tarea interdisciplinaria" (García, 1986: 69)

Lo que intentamos hacer ha sido solamente proponer hipótesis que deberían ser comprobadas en el campo para después llegar a conclusiones. Además, "ningún sistema global está dado en el punto de partida de la investigación. El sistema no está definido, pero es definible. Una definición adecuada sólo puede surgir en cada caso particular en el transcurso de la propia investigación" (García, 1986: 46).

\section{CONCLUSIONES}

Los distintos modelos en salud pública analizan su objeto de acuerdo con el pensamiento hegemónico vigente, los paradigmas van cambiando y surgen nuevos modelos para intentar interpretar los fenómenos de salud-enfermedad. Los modelos representan la forma teórica en que sus idealizadores y sus seguidores interpretan la realidad. Entre los modelos aceptados por el pensamiento científico del momento histórico que se está viviendo, creemos que no hay el más correcto, pero si aquél con el que el investigador más se identifique. Por lo tanto, este ensayo no pretende criticar o enaltecer determinados modelos, pero sí reflexionar sobre cuál se adapta mejor a nuestro problema y que esté de acuerdo con nuestra interpretación de la realidad.

Cada modelo tiene sus ventajas y limitaciones, los brotes bien analizados con el enfoque epidemiológico clásico (López, 1991) no tienen la visión integradora buscada, pero tiene otros puntos favorables como la buena sistematización de la información y el análisis de los aspectos clínicos y biomédicos bien desarrollados. Como ya se mencionó anteriormente, para investigaciones de rutina en servicios de salud nos parece satisfactorio, pero creemos que es insuficiente para una mayor comprensión del problema.
El brote de Boca da Mata investigado por nosotros establece relaciones, pero no establece la función de cada parte del problema porque no establece los subsistemas; tampoco profundiza en el estudio de algunos elementos considerados importantes para el entendimiento de la ocurrencia de los brotes. Al analizar el estudio hecho anteriormente, nos parece tener las ideas claras y llegar a conclusiones satisfactorias, principalmente porque nuestro objeto no es complejo, una buena investigación tiene enfoque sistémico, pero nuestro estudio pudiera tener más construcción intelectual.

Algunos estudios sobre "procesos de desarrollo» y las condiciones locales tanto ambientales como sociales, fueran desarrolladas por el grupo de investigádores asociados a la Federación Internacional de Institutos de Estudios Avanzados (IFIAS). Estos trabajos plantean como objetivo fundamental el estudio de las transformaciones en el medio físico/ biótico que la intervención humana provoca a través de sus actividades productivas (Tutela, 1989; García, 1988a, 1988b). Un análisis más profundo de estas investigaciones probablemente contribuirían para el desarrollo de nuestros estudios sobre el aparecimiento de brotes de rabia humana por murciélagos y los cambios en los procesos productivos locales.

Con el fin de tener una mayor construcción intelectual del objeto y no privilegiar un determinado aspecto del problema, en detrimento de otros, nos parece que la teoría de los sistemas complejos podría contribuir bastante. Este diseño de investigación desemboca en un trabajo interdisciplinario que nos parece muy atractivo. El único inconveniente es que esta teoría no se basa hasta el momento en estudios en el área de la salud con objetos semejantes al propuesto por nosotros, existiendo solamente estudios muy interesantes en el área de nutrición (Duval, 1986; García, 1982, 1984). Sin embargo, este pequeño problema es al mismo tiempo un desafío, porque nos parece muy atractivo tener la oportunidad de intentar contribuir con nuestro estudio, a la validación de una teoría con relación a distintos campos del conocimiento. 


\section{AGRADECIMIENTO}

Al Dr. Guy Duval de Centro de Investigaciones y Estudios Avanzados, por propiciar un estudio dirigido sobre la teoría de sistemas complejos permitiendo que fuera posible desarrollar este ensayo, pero siendo de mi responsabilidad la interpretación hecha de él. Al Conselho Nacional de Desenvolvimento Científico e Tecnológio por mi beca de doctorado.

\section{RESUMEN}

\section{SCHNEIDER, M. C. Reflexión sobre los Modelos para el Estudio de los Brotes de Rabia Humana por Murciélago. Cad. Saúde Públ., Rio de Janeiro, 11 (2): 291-304, abr/jun, 1995.}

El presente trabajo es una reflexión sobre cuál de los posibles modelos en salud pública contribuiría más para el entendimiento de la ocurrencia de brotes de rabia humana transmitida por murciélagos. En un primer momento se hace una breve revisión sobre los modelos más utilizados para analizar nuestro objeto de estudio. Posteriormente se presenta la investigación de un brote de rabia humana transmitida por murciélagos con base en el modelo epidemiológico social. Este mismo brote se analiza nuevamente utilizando como marco teórico la teoría de sistemas complejos, como forma de un ejercicio teórico-práctico para intentar una aproximación a esta teoría, como un posible modelo para estudiar el problema presentado.

Palabras Clave: Rabia; Murciélagos; Teoría de Sistemas Complejos; Salud Pública

\section{REFERENCIAS BIBLIOGRAFICAS}

ASTUDILLO, V.; DORA, F. \& SILVA, A., 1986. Ecosistemas y estrategias regionales de control de la fiebre aftosa. Aplicación al caso de Rio Grande do Sul, Brasil. Boletin del Centro Panamericano de Fiebre Aftosa, 52: 47-61.

BAER, G. M., 1975. Historia Natural de la Rabia. México: La Prensa Médica Mexicana.
BREILH, J. \& GRANADA, E., 1986. Investigado da Saúde na Sociedade; Guia Pedagógico Sobre um Novo Enfoque no Método Epidemiológico. Sáo Paulo: Instituto de SaúdelRio de Janeiro: Abrasco.

CASTELLANO, P. L., 1987. Sobre el concepto de salud-enfermedad. Un punto de vista epidemiológico. Cuadernos Médicos Sociales, 42: 15-24.

CINVESTAV (Centro de Investigaciones y Estudios Avanzados), sal.. La Sección de Metodología y Teoría de la Ciencia. México: CINVESTAV.

DUBOS, R., 1975. El medio y la enfermedad. In: El Espejismo de la Salud. Utopías, Progreso y Cambio biológico (R. Dubos), Vol. IV, pp. 107143, México: Fondo de Cultura Económica. (Colección Popular, 146)

DUVAL, G., 1986. A Methodological Approach for the Retrospective Diagnosis of Nutritional Status. The Mexican Case. Ginebra: UNRISD.

FRENK, J., 1979. Salud, educación y medicina: expectativas y limitaciones. Ciencia y Desarrollo, 26: 133-138.

1992. La nueva salud pública. In: Instituto Nacional de Salud Pública: Cinco Años de Vida 1987-1991, pp. 17-36, Cuernavaca: INSP.

GALVÃO, M. A. M., 1988. A Febre Maculosa Brasileira em Minas Gerais e seas Determinantes. Tese de Mestrado, Rio de Janeiro: Escola Nacional de Saúde Pública, Fundação Oswaldo Cruz.

GARCÍA, R. (Coord.), 1982. Drought and Man the 1972 Case History. Vol. 1 (Nature pleads not guilty); Vol. 2 (The constant catastrophe: Mainutrition, famine and drought); Vol. 3. (The roots of catastrophe), Londres: Pergamon Press. 1984. Food Systems and Society: A Conceptual and Methodological Challenge. Geneva: UNRISD.

1986. Conceptos básicos para el estudio de sistemas complejos. In: Los Problemas del Conocimiento y la Perspectiva Ambiental del Desarrollo (E. Leff, coord.), pp. 45-71. México: Siglo XXI.

1988a. Modernización en el Agro: ¿Ventajas Comparativas Para Quién? (El Caso di, los Cultivos Comerciales del Bajío). México: Centro de Investigaciones y Estudios Avanzado.

1988b. Deterioro Ambiental y Pobreza en la Abundancia Productiva (el Caso de la Comarca Lagunera). México: Centro de Investigaciones y Estudios Avanzados.

GREENHALL, A. \& SCHMIDT, U., 1989. Natural History of Vampire Bats. Boca Raton: CRC Press. 
KONOLSAISEN, J. F.; MOURA, F. B. P. \& DEL PRIETO, H., 1991. Relatório da Viagen a Cruzeiro doSul-AC: Investigação do Surto de Raiva Humana por Morcego. Relatório enviado a Coordenação do Programa Nacional de Profilaxia da Raiva. Brasília, DF: MS/FNS. (Mimeo.)

LAURELL, A. C.,1989. Social analysis of collective health in Latin America. Social Science and Medicine, 28: 1183-1191.

LEAVELL, H. R. \& CLARK, E. G., 1965. What is preventive medicine Levels of application of preventive medicine. In: Preventive Medicine for the Doctor in the Community: An Epidemiologic Approach (Leavell, H. R. \& Clark, E. G.), pp. 07-38, New York: MacGraw-Hill.

LILLIENFELD, A. M. \& LILLIENFELD, D. E., 1980. Laying the foudation: The epidemiologic approach to disease. In: Foudation of Epidemiology (Lillienfeld, A. M. \& Lillienfeld, D. E.), 2nd ed., pp. 03-22, New York: Oxford University Press.

LÓPEZ, A., 1991. Presentación del Brote Epidémico de Choque Madre de Dios (Perú). Anales: Reunión de Consulta sobre la Atención a Personas Expuestas a la Rabia Transmitidas por Vampiros. Washington: OPS. (Mimeo.)

MAC MAHON, B. \& PUGH, T., 1970. Epidemiology concepts of cause. In: Epidemiology: Principles and Methods (B. Mac Mahon, \& T. Pugh), pp. 17-27, Boston: Little, Brown and Company.

MORAES, A. C. R. \& COSTA, W. M., 1987. Valorização do espaço. In: Uma Geografia da Sociedade, 2ª ed., pp. 60-92, São Paulo: Hucitec.

MOREIRA, R. J., 1981. Tecnologia, Relações Sociais e Condições de Vida dos Trabalhadores Rurais. III Congresso Paulista de Agronomia. Painel: Produtividade-Tecnologia e Meio Ambiente, Campinas. (Mimeo.)

1982. Agricultura brasileira: os interesses em jogo no inicio dos anos 80. Reforma Agrária, 12: 03-16.

PAVLOVSKY, E. N., sal. Natural Nidality of Transmissible Diseases. Moscow: Peace Publishers.

PÉREZ TAMAYO, R., 1974. El concepto de enfermedad. In: Tres variaciones sobre la muerte y otros ensayos biomédicos, pp. 01-21, México: La Prensa Médica Mexicana.
PEREIRA-FILHO, M.,1990. Relatório sobre o Surto de Raiva Humana em Pintadas-BA. Brasília, DF: Coordenação do Programa Nacional de Profilaxia da Raiva, FNS-MS. (Mimeo.)

ROSEMBERG, F., 1986. Estructura social y epidemiología veterinaria en América Latina. Boletín del Centro Panamericano de la Fiebre Aftosa, 52: 03-23.

SAN MARTÍN, H. 1975. Funciones de la medicina social y la salubridad y el ambiente y la salud. In: Salud y Enfermedad, pp. 37-40, México: La Prensa Médica Mexicana.

SANTOS, M., 1986. Por uma Geografia Nova. $3^{\text {a }}$ ed., São Paulo: Hucitec.

SCHNEIDER, M. C., 1990. Estudo de Avaliação Sobre Área de Risco para a Raiva no Brasil. Tese de Mestrado, Rio de Janeiro:

1991. Situación Epidemiológica de la Rabia Trasmitida por Murciélagos en Brasil: El Brote de Apiacaí/MT. Reunión de Consulta sobre la Atención a Personas Expuestas a la Rabia Trasmitida por Vampiros, Anais, Washington, OPS. (Mimeo.)

SCHNEIDER, M. C. \& BURGOA, C. S., 1994. Tratamiento contra la rabia humana: Un poco de su historia. Revista de Saúde Pública, 28: 454-463.

SILVA, L. J., 1985. Organização do espaço e doença. In: Epidemiologia I, pp. 159-185, Rio de Janeiro: PEC-ENSP/Abrasco. (Coleção Textos de Apoio)

SUSSER, M., 1973. Agent, host and environment as an ecological system. In: Causal Thinking in the Health Sciences Concepts and Strategies of Epidemiology (M. Susser), pp. 26-47, New York: Oxford University Press.

TUTELA, F., 1989. La Modernización Forzada del Tropico: El Caso de Tabasco. Mexico: El Colegio de Mexico.

UIEDA, W., 1987. Morcegos hematófagos e a raiva dos herbívoros no Brasil. An. Ci. Fiube, Uberaba, 1: 13-29. 